\title{
LE TRASFORMAZIONI DELLE SUPERFICIE PER CONFIGURAZIONI INVARIABILI.
}

\author{
Memoria di Pietro Tortorici (Palermo) ${ }^{\mathrm{I}}$ ).
}

Adunanza del 27 novembre $192 \mathrm{I}$.

Se fra due superficie $S, S^{\prime}$ puó porsi una corrispondenza biunicoca di punto a punto tale che la configurazione costituita da due punti corrispondenti $P, P^{\prime} \mathrm{e}$ dai piani $\pi, \pi^{\prime}$, tangenti rispettivamente alle superficie in tali punti rimanga invariabile al variare della coppia di punti corrispondenti, diremo che le due superficie sono vincolate o sono trasformate una dell'altra per configurazioni invariabili.

Evidentemente se fra due superficie esiste un tale vincolo risultano pure trasformate per configurazioni invariabili due altre superficie ad esse rispettivamente parallele e del resto arbitrarie; neila ticerca dunque di tutte le coppie di superficie godenti della proprietà indicata è lecito sostituire a ciascuna di esse una superficie parallela.

Darboux, che appunto si è occupato di tale ricerca ${ }^{2}$ ), è pervenuto al seguente risultato:

Se due superficie sono vincolate per configurazioni invariabili e non sono parallele esse sono parallele rispettivamente o a due superficie a curvatura totale costañte ed uguale o a due superficie d'area minima.

Il caso delle superficie a curvatura totale costante è ben noto; per le trasformazioni delle superficie d'area minima il Branchr ha poi dato le effettive formule di trasformazione dimostrandole per verifica ${ }^{3}$ ).

I) Estratto dalla tesi di abilitazione dll'insegnamento presentata alla R. Scuola Normale Superiore di Pisa (aprile 1920).

2) Cfr. G. Darboux, Lȩons sur la théorie générale des surfaces t. III (Gauthier-Villdrs et fils, Paris I894), p. $44 \mathrm{I}$.

3) Cfr. L Brancht, Sulle trasformazioni di Darboux delle superficie di area minima [Atti del IVo Congresso Internazionale dei Matematıcı, Vol. II (Sez II, Geometria), (Roma, Tipografia della R. Açcadem1a dei Lincei, 1909)], pag 264. 
Scopo principale della presente ricerca è di ritrovare direttamente tali formule e studiare particolarmente le trasformazioni delle superficie minime alle quali dànno luogo; il problema però è qui ripreso dal principio con un metodo nuovo e sono messi in luce altri casi di trasformazione taciuti dal Darboux $i$ quali, benchè siano semplici e meno importanti di quelli citati, sono pure abbastanza interessanti.

La ricerca è divisa in due capitoli: nel primo sono esaminati tutti i possibili casi di trasformazione, nel secondo sono studiate le trasformazioni delle superficie d'area minima.

\section{CaPitolo I.}

\section{Le condizioni di trasformabilità ed $i$ casi possibili di trasformazione.}

1. Le due superficie $S, S^{\prime}$, luogo rispettivamente dei punti $P \equiv(x, y, z)$, $P^{\prime} \equiv\left(x^{\prime}, y^{\prime}, z^{\prime}\right)$, siano vincolate per configurazioni invariabili.

Siano le $x, y, z ; x^{\prime}, y^{\prime}, z^{\prime}$ funzioni, al solito, di due variabili indipendenti $u, v$ e indichiamo per la superficie $S$ con

$$
X_{1}, \quad Y_{1}, Z_{1} ; \quad X_{2}, \quad Y_{2}, Z_{2} ; \quad X_{3}, Y_{3}, Z_{3}
$$

i coseni direttori rispettivamente della tangente alla linea secondo cui $v$ è costante, della tangente alla linea secondo cui $u$ è costante e della normale alla superficie. Notazioni analoghe munite di apici valgano per la superficie $S^{\prime}$.

Essendo $\cos \gamma, \cos \beta, \cos \beta^{\prime} ; t$ quattro costanti, esprimiamo analiticamente il vincolo per configurazioni invariabili mediante le quattro relazioni:

$$
\left\{\begin{array}{c}
S\left(x^{\prime}-x\right)^{2}=t^{2}, \\
S\left(x^{\prime}-x\right) X_{3}=t \cos \beta, \quad S\left(x^{\prime}-x\right) X_{3}^{\prime}=t \cos \beta^{\prime}, \\
S X_{3} X_{3}^{\prime}=\cos \gamma .
\end{array}\right.
$$

Indicando ora con $k$ una costante e con $\varphi=p(u, v)$ una funzione da determinare, si sodisfa a tali relazioni ponendo:

$$
\begin{gathered}
x^{\prime}=x+t\left(X_{1} \operatorname{sen} \beta \cos \varphi+X_{2} \operatorname{sen} \beta \operatorname{sen} \varphi+X_{3} \cos \beta\right) \\
X_{3}^{\prime}=X_{1} \operatorname{sen} \gamma \cos (\varphi+k)+X_{2} \operatorname{sen} \gamma \operatorname{sen}(\varphi+k)+X_{3} \cos \gamma
\end{gathered}
$$

e analoghe per $y^{\prime}, z^{\prime} ; Y_{3}^{\prime}, Z_{3}^{\prime}$.

Con ciò resta stabilito anche il legame:

$$
\cos \beta^{\prime}=\operatorname{sen} \beta \operatorname{sen} \gamma \cos k+\cos \beta \cos \gamma .
$$

A sistema coordinato $u, v$ sulle superficie $S$ si assuma quello delle linee di curvatura; allora se

$$
d s^{2}=E d u^{2}+G d v^{2}
$$


è il quadrato dell'elemento lineare di $S$, detri $r_{1}$ ed $r_{2}$ i raggi principali di curvatura nel punto generico $P$, derivando le (2), tenute presenti formule ben note che per brevità non riportiamo, si ottengono le relazioni:

(5)

$$
\left\{\begin{array}{c}
\frac{\mathrm{I}}{t} \frac{\partial x^{\prime}}{\partial u}=X_{1}\left[\frac{\sqrt{E}}{t}-\left(\frac{\partial \varphi}{\partial u}-\frac{\mathrm{I}}{\sqrt{G}} \frac{\partial \sqrt{E}}{\partial v}\right) \operatorname{sen} \beta \operatorname{sen} \varphi+\frac{\sqrt{E}}{r_{2}} \cos \beta\right] \\
+X_{2}\left(\frac{\partial \varphi}{\partial u}-\frac{\mathrm{I}}{\sqrt{G}} \frac{\partial \sqrt{E}}{\partial v^{\prime}}\right) \operatorname{sen} \beta \cos \varphi-X_{3} \frac{\sqrt{E}}{r_{2}} \operatorname{sen} \beta \cos \varphi \\
\frac{\mathrm{I}}{t} \frac{\partial x^{\prime}}{\partial v}=X_{1}\left(\frac{\partial \varphi}{\partial v}+\frac{\mathrm{I}}{\sqrt{E}} \frac{\partial \sqrt{G}}{\partial u}\right) \operatorname{sen} \beta \operatorname{sen} \gamma \\
+X_{2}\left[\frac{\sqrt{G}}{t}+\left(\frac{\partial \varphi}{\partial v}+\frac{\mathrm{I}}{\sqrt{E}} \frac{\partial \sqrt{G}}{\partial u}\right) \operatorname{sen} \beta \cos \varphi+\frac{\sqrt{G}}{r_{1}} \cos \beta\right]-X_{3} \frac{\sqrt{G}}{r_{1}} \operatorname{sen} \beta \operatorname{sen} \varphi .
\end{array}\right.
$$

Per esprimere che la superficie $S^{\prime}$, luogo del punto $P^{\prime} \equiv\left(x^{\prime}, y^{\prime}, z^{\prime}\right)$, ha per coseni direttori della normale in $P^{\prime}$ le $X_{j}^{\prime}, Y_{3}^{\prime}, Z_{3}^{\prime}$ imponiamo le due condizioni:

$$
\mathrm{S} X_{3}^{\prime} \frac{\partial x^{\prime}}{\partial u}=0, \quad \mathrm{~S} X_{;}^{\prime} \frac{\partial x^{\prime}}{\partial v}=\mathrm{o}
$$

le quali, per le precedenti, scrivonsi:

(6)

$$
\left\{\begin{array}{c}
\left(\frac{\partial \varphi}{\partial u}-\frac{1}{\sqrt{G}} \frac{\partial \sqrt{E}}{\partial v}\right) \operatorname{sen} \beta \operatorname{sen} \gamma \operatorname{sen} k \\
=\frac{\sqrt{E}}{r_{2}}[\operatorname{sen} \beta \cos \gamma \cos \varphi-\cos \beta \operatorname{sen} \gamma \cos (\varphi+k)]-\frac{\sqrt{E}}{t} \operatorname{sen} \gamma \cos (\varphi+k), \\
\left(\frac{\partial \varphi}{\partial v}+\frac{1}{\sqrt{E}} \frac{\partial \sqrt{G}}{\partial u}\right) \operatorname{sen} \beta \operatorname{sen} \gamma \operatorname{sen} k \\
=\frac{\sqrt{G}}{r_{1}}[\operatorname{sen} \beta \cos \gamma \operatorname{sen} \varphi-\cos \beta \operatorname{sen} \gamma \operatorname{sen}(\varphi+k)]-\frac{\sqrt{G}}{t} \operatorname{sen} \gamma \operatorname{sen}(\varphi+k)
\end{array}\right.
$$

e costituiscono un sistema differenziale per la funzione incognita $\varphi$.

Le condizioni di integrabilità di tale sistema differenziale, in ordine e formule assai note della Geometria Infinitesimale e che non riportiamo nemmeno, scrivonsi:

(7)

$$
\left\{\begin{array}{c}
t^{2}\left(\operatorname{sen}^{2} \beta \operatorname{sen}^{2} \gamma \operatorname{sen}^{2} k+\operatorname{sen}^{2} \beta \cos ^{2} \gamma+\cos ^{2} \beta \operatorname{sen}^{2} \gamma-2 \operatorname{sen}_{1}^{2} \cos \beta \operatorname{sen} \gamma \cos \gamma \cos k\right) \frac{1}{r_{1} r_{2}} \\
+t \operatorname{sen} \gamma(\cos \beta \operatorname{sen} \gamma-\operatorname{sen} \beta \cos \gamma \cos k)\left(\frac{1}{r_{3}}+\frac{1}{r_{2}}\right)+\operatorname{sen}^{2} \gamma=0 .
\end{array}\right.
$$

Tale relazione esprime il teorema:

Perchè una superficie $S$ sia trasformabile per configurazioni invariabili è necessario e sufficiente che le sue due curvature totale e media siano vincolate da una relazione lineare. 
Se ponesi :

$$
\left\{\begin{array}{c}
\cos \gamma=c, \quad \cos \beta=b, \quad \cos \beta^{\prime}=b^{\prime}, \\
\frac{\mathrm{I}}{r_{\mathrm{I}} r_{2}}=K, \quad \frac{\mathrm{I}}{r_{1}}+\frac{\mathrm{I}}{r_{2}}=H,
\end{array}\right.
$$

la precedente relazione (7) scrivesi sotto la forma equivalente di Darboux:

$$
\left(1-b^{\prime 2}\right) t^{2} K+\left(b-b^{\prime} c\right) t H+\left(1-c^{2}\right)=0 .
$$

Per ragioni di simmetria, la curvatura totale $K^{\prime}$ e la curvatura media $H^{\prime}$ di $S^{\prime}$ saranno vincolate dalla relazione analoga:

$$
\left(1-b^{2}\right) t^{2} K^{\prime}+\left(b^{\prime}-b c\right) t H^{\prime}+\left(1-c^{2}\right)=0 .
$$

Esaminiamo ora $\mathrm{i}$ varì casi che possono presentarsi cominciando dai più semplici.

2. Se la relazione (9) è sodisfatta identicamente, se cioè si ha:

$$
c= \pm \mathrm{r}, \quad b^{\prime}= \pm b= \pm \mathrm{I}
$$

identica è pure la (IO) e la retta $P P^{\prime}$ congiungente due punti corrispondenti delle superficie $S, S^{\prime}$ è una loro normale comune: esse risultano quindi parallele.

Intendiamo escluso questo caso evidente di trasformazione. ha:

la (9) dà:

3. Se la superficie $S$ è una sviluppabile cioè se $K=0$ e nel medesimo tempo si

e la (10) fornisce:

$$
\mathrm{I}-c^{2}=\mathrm{o}, \quad c= \pm \mathrm{I},
$$

$$
b= \pm b^{\prime}
$$

$$
K^{\prime}=\text { o, }
$$

cioè: Anche la superficie $S^{\prime}$ è sviluppabile.

Allora, essendo in questo caso:

dalla (3) risulta:

(I I)

e le (6) diventano:

$$
\operatorname{sen} \gamma=0
$$

$$
X_{3}=X_{3}^{\prime}
$$

$$
\begin{aligned}
& \frac{\sqrt{E}}{r_{2}} \operatorname{sen} \beta \cos \varphi=0 \\
& \frac{\sqrt{G}}{r_{\mathrm{I}}} \operatorname{sen} \beta \operatorname{sen} \phi=0
\end{aligned}
$$

onde, posto $\frac{1}{r_{2}}=0$, deducesi $\varphi=0$ e perció le (2) danno:

$$
x^{\prime}=x+t\left(X_{1} \operatorname{sen} \beta+X_{3} \cos \beta\right)
$$

son le analoghe circolando. 
La (II) dimostra intanto che in due punti corrispondenti i piani tangenti a $S, S^{\prime}$ (osculatori ai rispettivi spigoli di regresso $C_{o}, C_{\mathrm{o}}^{\prime}$ ) sono paralleli e ciò esprime che:

Lo spigolo di regresso $C_{\mathrm{o}}^{\prime}$ della sviluppabile $S^{\prime}$ è una curva trasformata di Combescure dello spigolo di regresso $C_{0}$ della sviluppabile $S$.

Le equazioni della curva $C_{\circ}^{\prime}$ si trovano immediatamente.

Usiamo le notazioni solite ${ }^{4}$ ) apponendo l'indice zero agli elementi di $C_{0}$, l'indice zero e l'apice a quelli di $C_{\mathrm{o}}^{\prime}$. Se gli elementi di $C_{\mathrm{o}}$ sono espressi in funzione del suo arco $s_{\mathrm{o}}$ e se $u_{\mathrm{o}}$ è un parametro, pel punto generico $P \equiv(x, y, z)$ di $S$ puó porsi:

$$
x=x_{0}+u_{0} \alpha_{0}
$$

e analoghe, onde per quello $P^{\prime} \equiv\left(x^{\prime}, y^{\prime}, z^{\prime}\right)$ di $S^{\prime}$ si ha:

$$
x^{\prime}=x_{0}+u_{0} \alpha_{0}+t\left(X_{1} \operatorname{sen} \beta+X_{3} \cos \beta\right)=x_{0}+u_{0} \alpha_{0}+t\left(x_{0} \operatorname{sen} \beta+\lambda_{0} \cos \beta\right)
$$

e da questa traesi subito per lo spigolo di regresso $C_{\mathrm{o}}^{\prime}$ :

$$
x_{0}^{\prime}=x_{0}+t\left(\lambda_{0}-\frac{P_{0}}{T_{0}} \alpha_{0}\right) \cos \beta=x_{0}+b\left(\lambda_{0}-\frac{P_{0}}{T_{0}} \alpha_{0}\right) \quad(b \text { costante })
$$

con le analoghe e tali relazioni segnalano che:

La curva $C_{\mathrm{o}}^{\prime}$ è tracciata sulla sviluppabile rettificante di $C_{\mathrm{o}}$.

La relazione fra $C_{o}$ e $C_{o}^{\prime}$ essendo involutoria vale la cosa inversa: in altre parole $C_{o}^{\prime}$ è pure una geodetica sulla sviluppabile rettificante di $C_{\mathrm{o}}$.

Le circostanze geometriche di questo caso in esame restano fissate completamente dal seguente teorema:

Se sopra una sviluppabile $\widehat{S}$ si assumono due geodetiche $C_{\mathrm{o}}, C_{\mathrm{o}}^{\prime}$ vincolate da una trasformazione di Combescure, le due sviluppabili $S, S^{\prime}$ aderenti a $C_{0}, C_{o}^{\prime}$ possonsi riferire punto a punto, ed in una semplice infinita di modi, in modo che risultino vincolate per configurazioni invariabili.

Intanto l'ipotesi che le due geodetiche siano vincolate da una trasformazione di Combescure porta che la distanza dei loro piani osculatori in punti situati sopra una medesima generatrice di $\bar{S}$ è costante.

Poiche infatti le due geodetiche $C_{0}, C_{0}^{\prime}$ sono ugualmente inclinate sulle generatrici di $\bar{S}$, detto $\sigma$ tale angolo di inclinazione e ritenendo le notazioni solite per lo spigolo di regresso $\bar{C}$ di $\bar{S}$, luogo del punto $\bar{P} \equiv(\bar{x}, \bar{y}, \bar{z})$; per le coordinate dei punti

4) Cfr. L. BIANCHt, Lezioni di Geothetria differenziale (2 edizione) Val. 1, Cap. I (Pisa, Spoerrí, Igo2). 
$P_{\mathrm{o}} \equiv\left(x_{\mathrm{o}}, y_{\mathrm{o}}, z_{\mathrm{o}}\right), P_{\mathrm{o}}^{\prime} \equiv\left(x_{\mathrm{o}}^{\prime}, y_{0}^{\prime}, z_{\mathrm{o}}^{\prime}\right)$ di $C_{\mathrm{o}}, C_{\mathrm{o}}^{\prime}$ si ha com'è noto:

$$
\begin{array}{ll}
x_{0}=\bar{x}+t_{\mathrm{o}} \bar{\alpha}, \quad t_{\mathrm{o}}=\frac{\mathrm{I}}{\operatorname{sen} \sigma}\left[c_{\mathrm{o}}-\int \operatorname{sen} \sigma d \bar{s}\right] ; \quad\left(c_{\mathrm{o}} \text { costante }\right) \\
x_{\mathrm{o}}^{\prime}=\bar{x}+t_{\mathrm{o}}^{\prime} \bar{\alpha}, \quad t_{\mathrm{o}}^{\prime}=\frac{\mathrm{I}}{\operatorname{sen} \sigma}\left[c_{\mathrm{o}}^{\prime}-\int \operatorname{sen} \sigma d \bar{s}\right], \quad\left(c_{\mathrm{o}}^{\prime} \text { costante }\right)
\end{array}
$$

e le analoghe, $\bar{s}$ essendo l'arco di $\bar{C}$.

Allora, per il tratto $\tau$ di gencratrice di $\bar{S}$ intercetto fra $C_{0}$ e $C_{0}^{\prime}$, traesi:

$$
\tau=\frac{c_{\mathrm{o}}-c_{\mathrm{o}}^{\prime}}{\operatorname{sen} \sigma}=\frac{b}{\operatorname{sen} \sigma}, \quad(b \text { costante })
$$

e la distanza dei piani osculatori risulta appunto $b$.

È subito visto che puó scriversi :

$$
x_{0}^{\prime}=x_{0}+\tau \vec{x}=x_{0}+b\left(\lambda_{0}-\frac{P_{0}}{T_{0}} \alpha_{0}\right) .
$$

Osservando infine che due generatrici corrispondenti di $S, S^{\prime}$ sono parallele, presi su esse in modo arbitrario i punti $P \equiv(x, y, z), P^{\prime} \equiv\left(x^{\prime}, y^{\prime}, z^{\prime}\right)$, detta $t$ la loro distanza, pongasi :

$$
\frac{b}{t}=\cos \beta, \quad(\cos \beta \text { costante })
$$

e si stabilisca fra i punti di $S$ e $S^{\prime}$ la corrispondenza data dalle formule:

$$
x^{\prime}=x+t\left(x_{0} \operatorname{sen} \beta+\lambda_{0} \cos \beta\right)=x+t\left(X_{1} \operatorname{sen} \beta+X_{3} \cos \beta\right)
$$

e analoghe le quali dimostrano appunto completamente il teorema enunciato.

4. Supponendo ora che la (9) non sia identica poniamo:

talmente che essa scrivesi:

$$
K=\mathrm{o}, \quad \frac{\mathrm{I}}{r_{2}}=\mathrm{o}
$$

$$
-t\left(b-b^{\prime} c\right) \frac{\mathrm{I}}{r_{1}}=\mathrm{I}-c^{2}
$$

e mostra che la superficie $S$ è un cilindro circolare retto.

Essendo $I-c^{2} \neq 0$, alla superficie $S^{\prime}$ possiamo sostituire la superficie parallela per la quale risulti:

ossia :

$$
b^{\prime}=b c
$$

$$
\cos \beta^{\prime}=\cos \beta \cos \gamma
$$

Allora per la (4) deve essere:

$$
\cos k=\mathrm{o}, \quad \operatorname{sen} k=\mathrm{I}
$$


e la (IO) dd:

$$
K^{\prime}=-\frac{\mathrm{I}-c^{2}}{\left(\mathrm{I}-b^{2}\right) t^{2}}=-\frac{\operatorname{sen}^{2} \gamma}{t^{2} \operatorname{sen}^{2} \beta}
$$

e si riconosce cosi la possibilità di vincolare per configurazioni invariabili una superficie a curvatura nulla con una a curvatura totale costante.

Verifichiamo la cosa per mezzo delle formule generali.

Assumiamo per asse delle z l'asse del cilindro, per linee $v$ costante le generatrici, per $u$ costante le sue sezioni rette.

Detto $\rho$ il raggio dẹl cilindro conțato positivamente dall'asse verso la superficie, $\theta$ una variabile poniamo:

Si ha:

$$
\begin{aligned}
& x=\rho \cos \theta, \\
& y=p \operatorname{sen} \theta, \quad\left(\theta=\frac{v}{p}\right), \\
& z=u .
\end{aligned}
$$

$$
E=G=\mathrm{I}
$$

e, se per direzione positiva della tangente alle linee $u$ costante prendesi quella degli archi crescenti positivamente, bisognerà porre:

$$
\begin{array}{lll}
X_{1}=0, & Y_{1}=0, & Z_{1}=\mathrm{r}, \\
X_{2}=-\operatorname{sen} \theta, & Y_{2}=\cos \theta, & Z_{2}=0, \\
X_{3}=-\cos \theta, & Y_{3}=-\operatorname{sen} \theta, & Z_{3}=0 .
\end{array}
$$

Allora, conformemente a convenzioni fondamentali, bisognerà ritenere:

$$
p=-r_{1}=t \cos \beta
$$

c le (2), eseguita ogni riduzione, dànno:

$$
\left\{\begin{array}{l}
x^{\prime}=-t \operatorname{sen} \beta \operatorname{sen} \theta \operatorname{sen} \varphi, \\
y^{\prime}=t \operatorname{sen} \beta \cos \theta \operatorname{sen} \phi, \\
z^{\prime}=u+t \operatorname{sen} \beta \cos \varphi,
\end{array}\right.
$$

la funzione $p(u, v)$ sodisfacendo alle equazioni differenziali:

$$
\begin{aligned}
& \frac{\partial \varphi}{\partial u}=\frac{\mathrm{I}}{t \operatorname{sen} \beta} \operatorname{sen} \varphi, \\
& \frac{\partial \varphi}{\partial v}=-\frac{\mathrm{I}}{t \cos \beta} \operatorname{cotg} \gamma \operatorname{sen} \varphi
\end{aligned}
$$

alle quali appunto riduconsi le (6).

Tenute presenti le precedenti formule, per l'elemento lineare $d s^{\prime}$ di $S^{\prime}$ trovasi 
l'espressione:

$$
d s^{\prime 2}=\cos ^{2} \varphi d u^{2}+\frac{\operatorname{tg}^{2} \beta}{\operatorname{sen}^{2} \gamma} \operatorname{sen}^{2} \varphi d v^{2}
$$

e pei coefficienti $D_{1}, D_{1}^{\prime}, D_{1}^{\prime \prime}$ della suı seconda forma fondamentale trovasi:

$$
\begin{aligned}
& D_{1}=-\frac{\operatorname{sen} \gamma}{t \operatorname{sen} \beta} \operatorname{sen} \varphi \cos \varphi, \\
& D_{1}^{\prime}=0, \\
& D_{1}^{\prime \prime}=\frac{\operatorname{sen} \beta}{t \cos ^{2} \beta \operatorname{sen} \gamma} \operatorname{sen} \varphi \cos \varphi
\end{aligned}
$$

onde, per la curvatura totale $K^{\prime}$ di $S^{\prime}$, uttienesi ancora:

$$
K^{\prime}=-\frac{\operatorname{sen}^{2} \gamma}{t^{2} \operatorname{sen}^{2} \beta} \text {. }
$$

Introducendo la variabile $\theta$, poichè $v=\rho \theta$ e $\rho=-t \cos \beta$ si ha:

$$
d s^{\prime 2}=\cos ^{2} \varphi d u^{2}+t^{2} \frac{\operatorname{sen}^{2} \beta}{\operatorname{sen}^{2} \gamma} \operatorname{sen}^{2} \varphi d \theta^{2}
$$

e le equazioni alle quali sodisfa la funzione $\varphi(u, v)$ si scrivono:

$$
\left\{\begin{array}{l}
\frac{\partial \varphi}{\partial u}=\frac{1}{t \operatorname{sen} \beta} \operatorname{sen} \varphi, \\
\frac{\partial \phi}{\partial \theta}=\frac{\cos \gamma}{\operatorname{sen} \gamma} \operatorname{sen} \phi .
\end{array}\right.
$$

Ora si puó, senza restrizione, ritenere:

cioè

$$
\left|K^{\prime}\right|=\mathbf{I}
$$

(14)

Allora è :

$$
\left|\frac{\operatorname{sen}^{2} \gamma}{t^{2} \operatorname{sen}^{2} \beta}\right|=1 \text {. }
$$

$$
\begin{aligned}
& d s^{\prime 2}=\cos ^{2} \varphi d u^{2}+\operatorname{sen}^{2} \varphi d \theta^{2} \\
& \rho^{2}=t^{2} \cos ^{2} \beta=\frac{\cos ^{2} \beta \operatorname{sen}^{2} \gamma}{\cos ^{2} \beta} .
\end{aligned}
$$

$\mathrm{Si}$ vede subito che:

Le linee $u, \theta$ sono, sulla superficie $S^{\prime}$, le linee di curvatura.

Tanto risulta direttamente dal fatto che si ha:

$$
F^{\prime}=\mathrm{o}, \quad D_{\mathrm{s}}^{\prime}=\mathrm{o}
$$

ed anche dalla osservazione immediata cha la funzione $\phi$ sodisfa, in forza di (13), (14), 
alla equazione:

$$
\frac{\partial^{2} \varphi}{\partial u^{2}}-\varepsilon \frac{\partial^{2} \psi}{\partial v^{2}}=\operatorname{sen} \varphi \cos \varphi\left\{\frac{1}{\left\{t^{2} \operatorname{sen}^{2} \beta\right.}-\varepsilon \frac{\cos ^{2} \gamma}{\operatorname{sen}^{2} \gamma}\right\} . \quad(\varepsilon= \pm \mathrm{r})
$$

Se $\varepsilon=+\mathrm{I}$ questa scrivesi:

e si ha:

$$
\frac{\partial^{2} \varphi}{\partial u^{2}}-\frac{\partial^{2} \varphi}{\partial \theta^{2}}=\operatorname{sen} \varphi \cos \varphi
$$

$$
d s^{\prime 2}=\cos ^{2} \varphi d u^{2}+\operatorname{sen}^{2} \varphi d \theta^{2}
$$

mentre che, se $\varepsilon=-\mathbf{I}$, ponendo (per maggior chiarezza) $\varphi=i \lambda$ si ha:

$$
\frac{\partial^{2} \lambda}{\partial u^{2}}+\frac{\partial^{2} \lambda}{\partial \theta^{2}}=-\operatorname{sh} \lambda \operatorname{ch} \lambda
$$

e:

$$
d s^{2}=\operatorname{ch}^{2} \lambda d u^{2}+\operatorname{sh}^{2} \lambda d \theta^{2}
$$

delle quali espressioni $\dot{e}$ ben noto il significato dalla teoria delle superficie a curvatura costante ${ }^{5}$ ).

Riassumendo adunque: assunto un cilindro circolare retto di raggio $\rho$ avente l'asse coincidente con l'asse delle $z$ pongasi :

$$
\rho^{2}=b t^{2}=t^{2} \cos ^{2} \beta
$$

e, preso arbitrariamente $t$, si determini $b$ e quindi $\operatorname{sen}^{2} \beta$, indi $\operatorname{sen}^{2} \gamma$ da (14): per ogni soluzione $\varphi$ delle equazioni (13) le formule (I2) definiscono una superficie $S^{\prime}$ a curvatura costante $K= \pm \mathrm{I}$ sulla quale le linee $u, v$ sono le linee di curvatura e vincolata per configurazioni invariabili al cilindro $S$ di partenza.

Si scriverà:

$$
S^{\prime} \equiv S . C_{t}
$$

indicando simbolicamente con $C_{t}$ la trasformazione.

Siccome $\varphi$ contiene una costante arbitraria risulta:

Ogni cilindro circolare retto ammette una semplice infinità di superficie $S^{\prime}$ a curvatura totale costante contigue per trasformazione $C_{t}$.

5. Fermiamoci al caso in cui sia $\varepsilon=+\mathrm{I}$, cioè $K^{\prime}=-\mathrm{I}$ e consideriamo insieme la prima e la terza forma quadratica fondamentale della superficie trasformata $S^{\prime}$ :

$$
\begin{aligned}
& d s^{\prime 2}=E^{\prime} d u^{2}+G^{\prime} d \theta^{2}=\cos ^{2} \varphi d u^{2}+\operatorname{sen}^{2} \varphi d \theta^{2}, \\
& d s^{\prime \prime 2}=e^{\prime} d u^{2}+g^{\prime} d \theta^{2}=\operatorname{sen}^{2} \varphi d u^{2}+\cos ^{2} \varphi d \theta^{2} .
\end{aligned}
$$

5) Cfr. L, Bianchi, loc. cit. ${ }^{4}$ ), Vol. II, Cap. XXI, 
Tenuto conto delle equazioni alle quali sodisfa la funzione $\varphi(u, \theta)$ si ha

$$
\left\{\begin{array}{l}
\frac{\mathrm{I}}{\sqrt{e^{\prime} g^{\prime}}} \frac{\partial \sqrt{g^{\prime}}}{\partial u}=-\frac{\mathrm{I}}{t \operatorname{sen} \beta} \operatorname{tg} \varphi, \\
\frac{\mathrm{I}}{\sqrt{\bar{e}^{\prime} g^{\prime}}} \frac{\partial \sqrt{e^{\prime}}}{\partial \theta}=\operatorname{cotg} \gamma
\end{array}\right.
$$

e l'ultima di queste relazioni mostra che:

Sulla superficie $S^{\prime}$ le linee di curvatura lungo le quali $\theta$ è costante sono linee piane.

In ordine a teoremi noti si deduce allora:

Ogni superficie $S^{\prime}$ è una superficie di ENNEPER e conseguentemente anche una superficie della classe di JoAсhimsthal.

Le linee di curvatura lungo le quali la $u$ è costante sono allora sferiche; d'altra parte essendo:

$$
\frac{\mathrm{I}}{\sqrt{E^{\prime} G^{\prime}}} \frac{\partial \sqrt{G^{\prime}}}{\partial u}=\frac{\mathrm{I}}{t \operatorname{sen} \beta}
$$

è costante il raggio della sfera sulla quale è tracciata una qualunque di tali linee ed inoltre, sempre in forza della seconda delle (is), è costante l'angolo $\psi$ di inclinazione del piano di una linea $\theta$ sulla superficie poichè appunto si ha:

$$
\cot \psi=\frac{\mathrm{I}}{\sqrt{e^{\prime} g^{\prime}}} \frac{\partial \sqrt{e^{\prime}}}{\partial \theta}=\cot \gamma .
$$

La trasformazione è poi evidentemente reale e ricordando teoremi noti si deduce:

Le superficie $S^{\prime}$ sono elicoidi psendosferiche del Dint.

Aggiungiamo che, sempre restando nell'ipotesi $\varepsilon=+\mathrm{I}$, $\varphi$ è reale e se si vuole che anche le linee $u$ di curvatura siano piane basterà scrivere che la funzione $\varphi$ dipende solo da $u$ cioè :

$$
\frac{\partial \varphi}{\partial \theta}=0, \quad \cos \gamma=0 .
$$

In tali condizioni, per la curvatura geodetica di tali linee si ottiene:

$$
\frac{\mathrm{I}}{\rho_{u}^{\prime}}=-\frac{\mathrm{I}}{\operatorname{sen} \phi} \frac{\partial \varphi}{\partial u}=-\frac{\mathrm{I}}{t \operatorname{sen} \beta}
$$

ció che conferma ancora che esse sono sferiche e perciò sono circolari.

Si dimostra allora facilmente che la superficie $S^{\prime}$ è l'ordinaria pseudosfera e la curva trattrice meridiana ha per asintoto l'asse del cilindro $S$; questa considerazione puó poi servire a provare, ove non si deducesse già direttamente, che in ogni caso l'asse del cilindro $S$ è l'asse della superficie elicoidale $S^{\prime}$.

Come si debba stabilire la corrispondenza biunivoca di punto a punto quando 
siano dati un cilindro $S$ ed un elicoide del Dint coassiale è cosa geometricamente quasi evidente; anzi la osservazione diretta di tale circostanza geometrica ci ha indotti ad esporre questo facile studio.

Infine se $\varepsilon=-$ I è $K^{\prime}=+$ I; a causa del legame (14) fra le costanti $t$, sen $\beta$, sen $\gamma$ la trasformazione è necessariamente immaginaria. La superficie $S^{\prime}$ è una deformata della sfera e la configurazione $\left(S, S^{\prime}\right)$ è analoga alla precedente.

$\mathrm{Si}$ osservi ancora che, quando si restringa indefinitamente il cilindro, fmo a ridurlo al suo asse, le configurazioni esammate riduconsi in sostanza a quelle studiate dal Bianchi per le superficie a curvatura totale positiva o negativa, ma mentre nella trattazione del BIANCHI dette configurazioni si ottengono come casi limiti delle trasformazioni $B_{\sigma}$ delle superficie di ugual curvatura qui sono presentate come casi limiti della trasformazione di una superficie a curvatura nulla in una a curvatura costante ${ }^{6}$ ).

6. Tornando alle relazioni (9), (Io), se si ha ad un tempo:

$$
K=\mathrm{o}, \quad K^{\prime}=\mathrm{o}, \quad \mathrm{I}-c^{2} \neq \mathrm{o},
$$

esse riduconsi alle seguenti:

$$
\begin{aligned}
& \frac{\mathrm{t}}{r_{2}}\left(b-b^{\prime} c\right) t+\left(\mathrm{1}-c^{2}\right)=0, \\
& \frac{\mathrm{t}}{r_{2}^{\prime}}\left(b^{\prime}-b c\right) t+\left(1-c^{2}\right)=0
\end{aligned}
$$

ed $S, S^{\prime}$ sono quindi due cilindri circolari retti.

Facilmente dimostrasi che essi sono paralleli; fissati $i$ raggi $r_{2}, r_{2}^{\prime}$ si hanno due relazioni fra le quattro costanti $b, b^{\prime}, c, t$ e da qui risulta, come geometricamente è evidente, che fra $S, S^{\prime}$ la corrispondenza per configurazioni invariabili puó stabilirsi in una doppia infinita di modi.

7. Supponiamo ora che essendo

sia :

$$
K \neq \mathrm{o}, \quad K^{\prime} \neq \mathrm{o},
$$

$$
1-c^{2} \neq 0
$$

e che ancora le normali a $S, S^{\prime}$ in punti corrispondenti non siano complanari.

Allora alle superficie $S, S^{\prime}$ possonsi sostituire le superficie parallele, che continuiamo a chiamare $S, S^{\prime}$ e per le quali le relazioni (9) e (ro) sono prive dei secondi termini ciò che equivale a porre:

$$
b=b^{\prime}=0
$$

6) Cfr. L. BtanchI, loc. cit. ${ }^{4}$ ), Vol. Il, $\$ \$ 334,387,406$ 
In altre parole le superficie $S, S^{\prime}$ vengono sostituite dalle superficie rispettivamente parallele per i piedi della minima distanza fra due normali sghembe corrispondenti.

In tali condizioni risulta:

$$
K=K^{\prime}=-\frac{\mathrm{I}-\hat{\boldsymbol{t}}^{2}}{t^{2}}
$$

cioè le superficie $S, S^{\prime}$ hanno la medesima curvatura totale costante.

$\dot{E}$ il caso classico delle trasformazioni di BäckLund e dalle relazioni del $n^{0}$ I potrebbero facilmente dedursi le effettive formule di trasformazione.

8. Facciamo ora l'ipotesi che essendo sempre

$$
K \neq 0, \quad K^{\prime} \neq 0 ; \quad 1-c^{2} \neq 0
$$

le normali alle superficie $S, S^{\prime}$ in due punti corrispondenti siano complanari; allora fra le costanti $b, b^{\prime}, c$ passa la relazione:

$$
I-b^{2}-b^{\prime 2}-c^{2}=2 b b^{\prime} c
$$

che puó anche scriversi:

$$
\left(\mathrm{I}-c^{2}\right)\left(\mathrm{I}-b^{\prime 2}\right)=\left(b-b^{2} c\right)^{2} .
$$

Detti $r_{1}, r_{2}$ ancora i raggi principali di curvatura delle superficie $S$ la (9) puó scriversi :

$$
r_{2}\left[t\left(b-b^{\prime} c\right)+\left(1-c^{2}\right) r_{1}\right]+\left[t\left(b-b^{\prime} c\right) r_{1}+\left(1-b^{\prime 2}\right) t^{2}\right]=0
$$

e ponendo:

si ha:

$$
\frac{\mathrm{I}-c^{2}}{t\left(b-b^{\prime} c\right)}=\frac{t\left(b-b^{\prime} c\right)}{t^{2}\left(\mathrm{I}-b^{\prime 2}\right)}=\frac{\mathrm{I}}{\alpha} \quad(\alpha \text { costante })
$$

ossia :

$$
r_{2}\left(1+\frac{r_{1}}{\alpha}\right)+\left(r_{1}+\alpha\right)=0
$$

$$
\left(r_{1}+\alpha\right)\left(r_{2}+\alpha\right)=0
$$

onde se non è $r_{1}=-\alpha$, è $r_{2}=-\alpha$. Quindi:

La superficie $S$ è necessariamente canale.

Peraltro la relazione (16) trae seco l'altra:

$$
\left(1-c^{2}\right)\left(1-b^{2}\right)=\left(b^{\prime}-b c\right)^{2}
$$

onde facilmente concludesi che $S^{\prime} \dot{e}^{\circ}$ pure una superficie canale avente con $S$ l'asse in comune.

I raggi principali di curvatura costante $\rho$ e $\rho^{\prime}$ sono rispettivamente per le due su. perficie dati dalle espressioni:

$$
p=\frac{t\left(b-b^{\prime} c\right)}{\mathrm{I}-c^{2}}, \quad \rho^{\prime}=\frac{t\left(b^{\prime}-b c\right)}{\mathrm{I}-c^{2}}
$$


le due superficie sono bensi parallele ma la corrispondenza fra i loro punti non è necessariamente quella stabilita dalle loro normali comuni.

9. Infine se

$$
K \neq \mathrm{o}, \quad K^{\prime}=\mathrm{o}, \quad \mathrm{I}-c^{2} \neq \mathrm{o},
$$

le (9), (ro) possonsi scrivere:

$$
\begin{aligned}
& \left(1-b^{\prime 2}\right) t^{2}+t\left(b-b^{\prime} c\right)\left(r_{1}+r_{2}\right)=0 \\
& \left(1-b^{2}\right) t^{2}+t\left(b^{\prime}-b c\right)\left(r_{1}^{\prime}+r_{2}^{\prime}\right)=0
\end{aligned}
$$

e mostrano che le due superficie $S, S^{\prime}$ hanno costante la somma dei raggi principali di curvatura. Sostituendo ad esse le superficie d'area minima loro rispettivamente parallele, poichè le precedenti relazioni non devono avere nulli $\mathrm{i}$ coefficienti, dovrà aversi:

onde:

$$
c= \pm \mathrm{I}, \quad b^{\prime 2}=\mathrm{I}, \quad b^{2}=\mathrm{I}, \quad|b|=\left|b^{\prime} c\right|
$$

$$
b=-b^{\prime} c \text {. }
$$

Lo studio accurato di questo interessante caso sarà fatto nel Cap. II, prima però di occuparcene vogliamo ancora fare qualche osservazione sulle trasformazioni in esame.

ro. Poichè fra due sviluppabili ( $\mathrm{cfr} . \mathrm{n}^{\circ}$ 3) la trasformazione per configurazioni invariabll puó porsi in una semplice infinità di modi e fra due cilindri circolari retti coassiali in una doppia infinità di modi, ci si puó proporre di decidere, in generale, quando fra due superficie $S, S^{\prime}$ il vincolo per configurazioni invariabili possa stabilirsi in più di un modo senza cambiare però la posizione relativa delle due superficie.

Una ricerca in tal senso porta al seguente risultato: le due superficie $S, S^{\prime}$ possono essere o due sfere concentriche, o due superficie sviluppabili quali quelle esaminate $\mathrm{al}^{\circ} 3$, o due superficie canali coassiali; nel primo caso il vincolo per configurazioni invariabili puó porsi in due modi soltanto; nel secondo e terzo in una semplice infinitd di modi.

Nel caso particolare che le due superficie canali siano anche sviluppabili la corrispondenza può stabilirsi, come è stato gid detto, in una doppia infinità di modi.

I I. Infine se ci proponessimo di ricercare le superficie che possano restare in loro medesime per configurazioni invariabili, pensando una tale superficie come costituita da due superficie uguali sorrapposte e sostituendo ad una di esse una superficie parallela, facilmente perverremto al risultato seguente: una superficie $S$ che possa rimanere in sè medesima per configurazioni invariabili è necessariamente o una sfera o una sviluppabile o una superficie canale.

Se la superficie è sferica la corrispondenza che la lascia in sè medesima puó porsi solo in un modo facendo corrispondere ad ogni punto quello diametralmente opposto; se è sviluppabile la corrispondenza puó porsi in una semplice infinita di modi potendo ad un punto qualunque della superficie farne corrispondere un altro purchè della me- 
desima generatrice; infine se la superficie è canale la corrispondenza puó ancora stabilirsi in una semplice infinità di modi potendo far corrispondere due punti qualunque della superficie purchè appartenenti ad una medesima sezione piana normale all'asse.

Soltanto nel caso di un cilindro circolare retto (superficie canale e sviluppabile) la corrispondenza puó porsi in una doppia infinità di modi.

\section{Capitolo II.}

\section{Le trasformazioni di DARBOUX delle superficie d'area minima.}

12. Esaminiamo ora il caso accennato al $\mathfrak{n}^{\circ} 9$ del Capitolo precedente. In questo caso $\dot{e}$ :

$$
c= \pm \mathrm{I}, \quad b^{\prime 2}=b^{2}=\mathrm{r}, \quad b^{\prime}=-b^{\prime} c
$$

onde $\mathrm{i}$ possibili valori simultanei per le costanti $c, b, b^{\prime}$ sono dati dai seguenti quattro casi :
a)
$c=\mathrm{I}, \quad b=-\mathrm{I}, \quad b^{\prime}=\mathrm{I}$,
ß)
$\mathfrak{c}=\mathrm{I}$,
$b=\mathrm{I}$,
$b^{\prime}=-\mathrm{I}$,
$\gamma)$
$c=-\mathrm{I}$,
$b=\mathrm{I}$,
$b^{\prime}=\mathrm{I}$,
8)
$c=-\mathrm{I}, \quad b=-\mathrm{I}, \quad b^{\prime}=-\mathrm{I}$.

Se peró si ha riguardo alle condizioni (I) di invariabilità della configurazionè si vede che tali quattro casi riconduconsi uno all'altro cambiando eventualmente la direzione positiva della normale di $S^{\prime}$ o di quella di $S$ o di ambedue le superficie. Ci limiteremo pertanto a considerare il caso $\alpha$ ).

Per avere le effettive formule di trasformazione in questo caso non ci serviremo delle relazioni stabilite al $\mathrm{n}^{\circ}$ I ma useremo un procedimento diretto assai semplice, perchè le relazioni (6), (7) dovendo essere necessariamente:

$$
\operatorname{sen} \gamma=0, \quad \operatorname{sen} \beta=0,
$$

anche supponendo che le costanti $\gamma, \beta, k, t$ siano complesse, diventano identiche e per la funzione incognita $?$ non potremmo trarre da esse le equazioni differenziali che ci necessitano.

Abbiamo già osservato che la superficie $S$ è una superficie d'area minima, riferendola alle sue linee di curvatura $u, v$ il suo elemento lineare ha l'espressione:

$$
d s^{2}=e^{2 \theta}\left(d u^{2}+d v^{2}\right)
$$

$\theta=\theta(u, v)$ essendo un integrale della equazione di LiouvilLe:

$$
\frac{\partial^{2} \theta}{\partial u^{2}}+\frac{\partial^{2} \theta}{\partial v^{2}}=e^{-2 \theta}
$$


Si hanno allora le formule:

(19)

$$
\begin{cases}\frac{\partial x}{\partial u}=e^{\theta} X_{1}, & \frac{\partial x}{\partial v}=e^{\theta} X_{2}, \\ \frac{\partial X_{1}}{\partial u}=-\frac{\partial \theta}{\partial v} X_{2}-e^{-\theta} X_{;}, & \frac{\partial X_{1}}{\partial v}=\frac{\partial \theta}{\partial u} X_{2}, \\ \frac{\partial X_{2}}{\partial u}=\frac{\partial \theta}{\partial v} X_{1}, & \frac{\partial X_{2}}{\partial v}=-\frac{\partial \theta}{\partial u} X_{1}+e^{-\theta} X_{3}, \\ \frac{\partial X_{i}}{\partial u}=e^{-\theta} X_{1}, & \frac{\partial X_{3}}{\partial v}=-e^{-\theta} X_{2} .\end{cases}
$$

Per le coordinate $x^{\prime}, y^{\prime}, z^{\prime}$ del punto generico $P^{\prime}$ di $S^{\prime}$ e per i coseni direttori $X_{3}^{\prime}, Y_{3}^{\prime}, Z_{j}^{\prime}$ della sua normale in $P^{\prime}$ pongasi:

$$
\left\{\begin{array}{l}
x^{\prime}=x+\left(L X_{1}+M X_{2}+N X_{j}\right) t^{2}, \quad(t \text { costante }) \\
X_{3}^{\prime}=P X_{1}+Q X_{2}+R X_{3}
\end{array}\right.
$$

con le analoghe circolando, $L, M, N ; P, Q, R$ essendo sei funzioni incognite di $u, v$.

Le condizioni di invariabilità della configurazione dei piani tangenti e dei punti corrispondenti di contatto, che in questo caso scriviamo sotto la forma:

$$
\begin{gathered}
\mathrm{S}\left(x^{\prime}-x\right)^{2}=t^{\prime}, \\
\mathrm{S} X_{3}\left(x^{\prime}-x\right)=-t^{2}, \quad \mathrm{~S} X_{3}^{\prime}\left(x^{\prime}-x\right)=t^{2}, \\
\mathrm{~S} X_{3} X_{3}^{\prime}=\mathrm{I},
\end{gathered}
$$

dànno per tali funzioni incognite le condizioni:

$$
\begin{gathered}
N=-\mathrm{I}, \quad R=\mathrm{I}, \\
L^{2}+M^{2}=\mathrm{o}, \quad P^{2}+Q^{2}=\mathrm{o}, \quad L P+M Q=2 .
\end{gathered}
$$

Posto allora:

$$
L=\sigma(u, v)
$$

e fissando, a causa della prima delle (2 I):

deducesi dalle altre:

$$
M=+i \sigma
$$

e quindi le (20) diventano:

$$
P=\frac{\mathrm{I}}{\sigma}, \quad Q=-\frac{i}{\sigma}
$$

$$
\begin{gathered}
x^{\prime}=x+t^{2}\left(\sigma X_{1}+i \sigma X_{2}-X_{3}\right) \\
X_{3}^{\prime}=\frac{1}{\sigma} X_{1}-\frac{i}{\sigma} X_{2}+X_{3} .
\end{gathered}
$$


Per le formule del quadro (19) le solite condizioni:

scrivonsi allora:

$$
\mathrm{S} X_{3}^{\prime} \frac{\partial x^{\prime}}{\partial u}=\mathrm{o}, \quad \mathrm{S} X_{3}^{\prime} \frac{\partial x^{\prime}}{\partial v}=\mathrm{o}
$$

$$
\begin{array}{r}
\frac{1}{\sigma} e^{\theta}+2 t^{2} \frac{\partial \log \sigma}{\partial u}+2 i t^{2} \frac{\partial \theta}{\partial v}-t^{2} e^{-\theta}\left(\sigma+\frac{1}{\sigma}\right)=0, \\
-\frac{i}{\sigma} e^{\theta}+2 t^{2} \frac{\partial \log \sigma}{\partial v}-2 i t^{2} \frac{\partial \theta}{\partial u}-i t^{2} e^{-\theta}\left(\sigma+\frac{1}{\sigma}\right)=0 .
\end{array}
$$

Per ottenere le effettive formule di trasformazione sotto l'aspetto di BIANCHI pongasi :

e le precedenti diventano:

$$
\sigma=\frac{e^{\theta^{\prime}}}{t}
$$

$$
\left\{\begin{array}{l}
\frac{\partial \theta^{\prime}}{\partial u}+i \frac{\partial \theta}{\partial v}=\frac{e^{\theta^{\prime}-\theta}-e^{-\left(\theta^{\prime}-\theta\right)}}{2 t}+\frac{t}{2} e^{-\left(\theta^{\prime}+\theta\right)} \\
i \frac{\partial \theta^{\prime}}{\partial v}+\frac{\partial \theta}{\partial u}=\frac{e^{\theta^{\prime}-\theta}-e^{-\left(\theta^{\prime}-\theta\right)}}{2 t}-\frac{t}{2} e^{-\left(\theta^{\prime}+\theta\right)}
\end{array}\right.
$$

mentre le (20) si scrivono:

$$
\left\{\begin{array}{l}
x^{\prime}=x+t e^{\theta^{\prime}}\left(X_{\mathrm{I}}+i X_{2}\right)-t^{2} X_{i}, \\
X_{3}^{\prime}=e^{-\theta^{\prime}}\left(X_{1}-i X_{2}\right)+X_{3} .
\end{array}\right.
$$

Ora, in forza della equazione (18), il sistema (22) è illimitatamente integrabile per $\theta^{\prime}$ ed il suo integrale generale, contenente oltre $t$ una costante arbitraria, soddisfa alla equazione :

$$
\frac{\partial^{2} \theta^{\prime}}{\partial u^{2}}+\frac{\partial^{2} \theta^{\prime}}{\partial v^{2}}=e^{-\theta^{\prime}} .
$$

L'elemento lineare della superficie $S^{\prime}$ luogo del punto $P^{\prime} \equiv\left(x^{\prime}, y^{\prime}, z^{\prime}\right)$ ha l'espressione:

e poichè inoltre si ha:

$$
d s^{\prime 2}=e^{2 \theta^{\prime}}\left(d u^{2}+d v^{2}\right)
$$

$$
\frac{\partial x^{\prime}}{\partial u}=e^{2 \theta^{\prime}} \frac{\partial X_{3}^{\prime}}{\partial u}, \quad \frac{\partial x^{\prime}}{\partial v}=-e^{2 \theta^{\prime}} \frac{\partial X_{3}^{\prime}}{\partial v},
$$

concludesi che la $S^{\prime}$ è una superficie d'area minima (immaginaria se $S$ è reale) vincolata alla $S$ per configurazioni invariabili.

Diremo con Bianchi che la $S^{\prime}$ è dedotta dalla $S$ mediante una trasformazione $D_{t}$ 
di Dafboux e scriveremo simbolicamente:

$$
S . D_{t} \equiv S^{\prime} .
$$

Contenendo la funzione $\theta^{\prime}$ una costante arbitraria si ha:

Ad ogni superficie minima $S$ sono contigue per trasformazione $D_{t}$ di Darboux una semplice infinità di superficie minime $S^{\prime}$.

Dalle formule precedenti deducesi inoltre:

Le trasformazioni di Darboux delle superficie d'area minima conservano le linee di curvatura e le linee di lunghezza nulla.

13. Tenendo presenti il sistema (22) e le formule (23) si trovano le relazioni:

(24)

$$
\left\{\begin{array}{r}
\frac{\partial x^{\prime}}{\partial u}=X_{1}\left[\frac{\mathrm{I}}{2} e^{\theta}-\frac{\mathrm{I}}{2} t^{2} e^{-\theta}+\frac{\mathrm{I}}{2} e^{2 \theta^{\prime}-\theta}\right] \\
+i X_{2}\left[-\frac{\mathrm{I}}{2} e^{\theta}+\frac{\mathrm{I}}{2} t^{2} e^{-\theta}+\frac{\mathrm{I}}{2} e^{2 \theta^{\prime}-\theta}\right]-X_{3} t e^{\theta^{\prime}-\theta} \\
\frac{\partial x^{\prime}}{\partial v}=i X_{1}\left[\frac{\mathrm{I}}{2} e^{\theta}+\frac{\mathrm{I}}{2} t^{2} e^{-\theta}-\frac{\mathrm{I}}{2} e^{2 \theta^{\prime}-\theta}\right] \\
+X_{2}\left[\frac{\mathrm{I}}{2} e^{\theta}+\frac{\mathrm{I}}{2} t^{2} e^{-\theta}+\frac{\mathrm{I}}{2} e^{2 \theta^{\prime}-\theta}\right]+i X_{3} t e^{\theta^{\prime}-\theta}
\end{array}\right.
$$

per mezzo delle quali deducesi l'identita:

$$
\frac{e^{\theta}}{t}\left(X_{1}^{\prime}+i X_{2}^{\prime}\right)+X_{3}^{\prime}=\frac{e^{\theta^{\prime}}}{t}\left(X_{1}+i X_{2}\right)-X_{3}
$$

la quale poteva scriversi direttamente osservando che per la coppia di superficie $S^{\prime}, S$ valgono le uguaglianze $\beta$ ) del $n^{\circ}$ precedente e che invece, cosi dedotta, conferma appunto tale circostanza.

14. Indicheremo brevemente il sistema (22) con la notazione $\Phi\left(\theta, \theta^{\prime} ; t\right)$ mettendo in evidenza prima la soluzione $\theta$ della equazione di Ltouville corrispondente alla superficie $S$ di cui si parte, indi quella $\theta^{\prime}$ corrispondente alla superficie $S^{\prime}$ cui si perviene mediante una trasformazione di Darboux a costante $t$.

Ció posto sia $S$ una superficie minima (reale o no) corrispondente alla soluzione $\theta$ e, corrispondentemente ai due sistemi $\Phi(\theta, \varphi ; a), \Phi(\theta, \lambda ; b)$, consideriamo le due superficie minime:

$$
S_{\mathrm{t}}^{\prime} \equiv S . D_{a}, \quad S_{\mathrm{a}}^{\prime} \equiv S . D_{b}
$$

e vediamo in che posizione relativa stanno nello spazio.

Se $P_{1} \equiv\left(x_{1}, y_{1}, z_{1}\right), P_{2} \equiv\left(x_{2}, y_{2}, z_{2}\right)$ sono i punti rispettivamente di $S_{1}^{\prime}, S_{2}^{\prime}$ corrispondenti ad un medesimo $P \equiv(x, y, z)$ di $S$; se con $X_{3,1}^{\prime}, Y_{3,1}^{\prime}, Z_{3,1}^{\prime} ; X_{3,2}^{\prime}, Y_{3,2}^{\prime}$, $Z_{j, 2}^{\prime}$ indichiamo $i$ coseni direttori della normale a $S_{1}^{\prime}, S_{z}^{\prime}$ in $P_{1}, P_{z}$ rispettivamente, si 
ha, secondo quanto precede:

$$
\left\{\begin{aligned}
x_{1} & =x+a e^{\varphi}\left(X_{1}+i X_{2}\right)-a^{2} X_{3}, \\
X_{3,1}^{\prime} & =a e^{-\varphi}\left(X_{1}-i X_{2}\right)+X_{3} \\
x_{2} & =x+b e^{2}\left(X_{1}+i X_{2}\right)-b^{2} X_{3} \\
X_{3,2}^{\prime} & =b e^{-\lambda}\left(X_{1}-i X_{2}\right)+X_{3}
\end{aligned}\right.
$$

con le analoghe. Da queste, ponendo:

$$
\left\{\begin{array}{l}
\alpha=\frac{a e^{\varphi}-b e^{\lambda}}{a^{2}-b^{2}}\left(X_{1}+i X_{2}\right)-X_{3}, \\
\beta=\frac{a e^{\varphi}-b e^{\lambda}}{a^{2}-b^{2}}\left(Y_{1}+i Y_{2}\right)-Y_{3}, \\
\gamma=\frac{a e^{\varphi}-b e^{\lambda}}{a^{2}-b^{2}}\left(Z_{1}+i Z_{2}\right)-Z_{3},
\end{array}\right.
$$

deduconsi subito le uguaglianze :

$$
\left\{\begin{array}{c}
\mathrm{S}\left(x_{\mathrm{r}}-x_{2}\right)^{2}=\left(a^{2}-b^{2}\right)^{2}, \\
\mathrm{~S} \alpha X_{3 \mathrm{I}}^{\prime}=\frac{\mathrm{I}}{a^{2}-b^{2}}\left(a^{2}-2 a b e^{\lambda-\varphi}+b^{2}\right), \\
\mathrm{S} \propto X_{3,2}^{\prime}=-\frac{\mathrm{I}}{a^{2}-b^{2}}\left(a^{2}-2 a b e^{\varphi-\lambda}+b^{2}\right), \\
\mathrm{S} X_{3,1}^{\prime} X_{3,2}^{\prime}=\mathrm{I} .
\end{array}\right.
$$

La prima di queste ultime esprime intanto che:

Se due superficie minime $S_{1}^{\prime}, S_{2}^{\prime}$ sono contigue per trasformazioni $D_{a}, D_{b}$ di Darboux ad una medesima superficie minima $S$, due punti $P_{1}, P_{2}$ di $S_{1}^{\prime}, S_{2}^{\prime}$ corrispondenti ad un medesimo punto $P$ di $S$ banno distanza costante.

In particolare si ha:

Due superficie minime contigue per trasformazione $D_{t}$ ad una medesima superficie minima $S$ hanno sempre nulla la distanza dei punti corrispondenti ad un medesimo di $S$.

15. Da quel che precede risulta che $S$ essendo una superficie immaginaria $S_{z}^{\prime}$ puó essere reale. Ricerchiamo se pure reale puó essere $S_{2}^{\prime}$.

Nell'ipotesi affermativa poichè

deducesi :

$$
\mathrm{S} X_{3,1}^{\prime} X_{3,2}^{\prime}=\mathbf{I}
$$

$$
X_{3,1}^{\prime}=X_{j, 2}^{\prime}
$$

e analoghe; e allora dalle (26), (27) per confronto si ottiene:

$$
\begin{gathered}
a e^{-\varphi}=b e^{-\lambda} \\
\lambda-\varphi=\log \frac{b}{a}, \quad \lambda=\varphi+\log \frac{b}{a}
\end{gathered}
$$


e se $\lambda$ è soluzione dell'equazione di Liouville non lo è $\varphi$ a meno che non sia $b=a$ nel qual caso $S_{1}^{\prime}$ ed $S_{2}^{\prime}$ non sono distinte. Dunque:

Da una superficie minima reale, mediante due successive trasformazioni di DaRBOUx, si perviene sempre ad una superficie minima immaginaria.

Questa proposizione puó anche esprimersi:

Le superficie minime contigue per trasformazioni di DaRBoux ad una medesima superficie immaginaria sono tuitte immaginarie eccezione fatta al piu per una di esse che può essere reale.

16. D'ora in avanti indicheremo con $S_{\theta}$ la superficie minima corrispondente alla soluzione $\theta$ dell'equazione di Liouvilue. Stabiliamo le formule di composizione delle trasformazioni di Darboux. Sia

e ancora

$$
S_{\varphi}^{\prime} \equiv S_{0} . D_{a}
$$

e siano

$$
S_{\psi}^{\prime \prime} \equiv S_{\varphi}^{\prime} \cdot D_{b} \equiv\left(S_{\theta} \cdot D_{a}\right) \cdot D_{b},
$$

$$
P \equiv(x, y, z), \quad P^{\prime} \equiv\left(x^{\prime}, y^{\prime}, z^{\prime}\right), \quad P^{\prime \prime} \equiv\left(x^{\prime \prime}, y^{\prime \prime}, z^{\prime \prime}\right)
$$

punti corrispondenti ordinatamente su $S_{\theta}, S_{\phi}^{\prime}, S_{\psi}^{\prime \prime}$.

Si hanno formule del tipo:

inoltre :

$$
\begin{aligned}
& x^{\prime}=x+a e^{\varphi}\left(X_{1}+i X_{2}\right)-a^{2} X_{3}, \\
& x^{\prime \prime}=x^{\prime}+b e^{\psi}\left(X_{1}^{\prime}+i X_{2}^{\prime}\right)-b^{2} X_{3}^{\prime} ;
\end{aligned}
$$

$$
X_{\mathrm{s}}^{\prime}=e^{\varphi} \frac{\partial X_{3}^{\prime}}{\partial u}, \quad X_{z}^{\prime}=-e^{\varphi} \frac{\partial X_{3}^{\prime}}{\partial v}
$$

con

$$
X_{3}^{\prime}=a e^{-\varphi}\left(X_{s}-i X_{2}\right)+X_{3} .
$$

Tenendo presenti tali formule ed il sistema fondamentale (22) di trasformazione si perviene, mediante sostituzione, alle espressioni seguenti:

con

$$
\begin{aligned}
& x^{\prime \prime}=x+A X_{1}+B X_{2}+C X_{3}, \\
& y^{\prime \prime}=y+A Y_{1}+B Y_{2}+C Y_{3}, \\
& z^{\prime \prime}=z+A Z_{1}+B Z_{2}+C Z_{3},
\end{aligned}
$$

$$
\begin{aligned}
& A=a\left(e^{\phi}-b^{2} e^{-\varphi}\right)+b\left(e^{\psi+\varphi-\theta}-a^{2} e^{\psi-\phi-\theta}\right), \\
& B=i a\left(e^{\phi}+b^{2} e^{-\phi}\right)+i b\left(e^{\psi+\varphi-\theta}-a^{2} e^{\psi-\phi-\theta}\right), \\
& C=-a^{2}-2 a b e^{\psi-\theta}-b^{2}
\end{aligned}
$$

le quali fanno appunto passare direttamente dalle superficie $S_{6}$ alle $S_{\psi}^{\prime \prime}$.

Se nelle formule ora scritte ponesi $\psi=\theta, b=-a$, si ha

$$
A=B=C=\mathrm{o}
$$


e si verifica cosi che

$$
\left(S_{\theta} \cdot D_{a}\right) \cdot D_{-a} \equiv S_{\theta}
$$

come era evidente a priori.

Medesimamente ottengonsi le formule:

con

$$
\begin{aligned}
& X_{3}^{\prime \prime}=M X_{1}+N X_{2}+X_{i}, \\
& Y_{3}^{\prime \prime}=M Y_{1}+N Y_{2}+Y_{3}, \\
& Z_{3}^{\prime \prime}=M Z_{1}+N Z_{2}+Z_{3}
\end{aligned}
$$

$$
\begin{aligned}
& M=b e^{\theta-\varphi-\psi}+a e^{-\varphi} \\
& N=i M
\end{aligned}
$$

e pure queste per $\varphi=0, b=-a$ dànno:

$$
X_{3}^{\prime \prime}=X_{3}, \quad Y_{3}^{\prime \prime}=Y_{3}, \quad Z_{3}^{\prime \prime}=Z_{3} .
$$

17. Allo scopo di stabilire un teorema notevole sulle trasformazioni in esame, premettiamo qui qualche considerazione analitica. Ferme restando le notazioni del $\mathrm{n}^{\circ}$ i 4 consideriamo i due sistemi $\Phi(\varphi, \theta ; a), \Phi(\lambda, \theta ; b)$ aventi in comune la soluzione $\theta$ dell'equazione di Liouville. Inoltre, per brevità, essendo $p, q$ due funzioni di $u, v$ e $k$ una costante poniamo:

$$
\begin{aligned}
& \frac{e^{p-q}-e^{-p+q}}{2 k}+\frac{k}{2} e^{-p-q}=A(p, q, k), \\
& \frac{e^{p-q}-e^{-p+q}}{2 k}-\frac{k}{2} e^{-p-q}=B(p, q, k) .
\end{aligned}
$$

Allora la funzione $\theta$ è soluzione comune ai due sistemi:

(29)

$$
\begin{aligned}
& \left\{\begin{array}{l}
\frac{\partial \varphi}{\partial u}+i \frac{\partial \theta}{\partial v}=A(\varphi, \theta, a), \\
i \frac{\partial \varphi}{\partial v}+\frac{\partial \theta}{\partial u}=B(\varphi, \theta, a),
\end{array}\right. \\
& \left\{\begin{array}{l}
\frac{\partial \lambda}{\partial u}+i \frac{\partial \theta}{\partial v}=A(\lambda, \theta, b), \\
i \frac{\partial \lambda}{\partial v}+\frac{\partial \theta}{\partial u}=B(\lambda, \theta, b) .
\end{array}\right.
\end{aligned}
$$

Ricerchiamo se possa esistere una soluzione comune $\psi$ ai due sistemi $\Phi(\varphi, \psi ; b)$, $\Phi(\lambda, \psi ; a)$. Ammessa per un momento vera la cosa devono essere sodisfatte le re- 
lazioni :

$$
\begin{aligned}
& \left\{\begin{array}{l}
\frac{\partial \varphi}{\partial u}+i \frac{\partial \psi}{\partial v}=A(\varphi, \psi, b), \\
i \frac{\partial \varphi}{\partial v}+\frac{\partial \psi}{\partial u}=B(\varphi, \psi, b),
\end{array}\right. \\
& \left\{\begin{array}{l}
\frac{\partial \lambda}{\partial u}+i \frac{\partial \psi}{\partial v}=A(\lambda, \psi, a), \\
i \frac{\partial \lambda}{\partial v}+\frac{\partial \psi}{\partial u}=B(\lambda, \psi, a) .
\end{array}\right.
\end{aligned}
$$

Da (29), (30), (3I), (32) deducesi :

$$
\begin{aligned}
& A(\varphi, \theta, a)-\frac{\partial \varphi}{\partial u}=A(\lambda, \theta, b)-\frac{\partial \lambda}{\partial u}, \\
& B(\varphi, \theta, a)-i \frac{\partial \varphi}{\partial v}=B(\lambda, \theta, b)-i \frac{\partial \lambda}{\partial v} \\
& A(\varphi, \psi, b)-\frac{\partial \varphi}{\partial u}=A(\lambda, \psi, a)-\frac{\partial \lambda}{\partial u}, \\
& B(\varphi, \psi, b)-i \frac{\partial \varphi}{\partial v}=B(\lambda, \psi, a)-i \frac{\partial \lambda}{\partial v},
\end{aligned}
$$

le quali forniscono le altre:

$$
\begin{aligned}
& A(\varphi, \theta, a)-A(\lambda, \theta, b)=A(\varphi, \psi, b)-A(\lambda, \psi, a), \\
& B(\varphi, \theta, a)-B(\lambda, \theta, b)=B(\varphi, \psi, b)-B(\lambda, \psi, a) .
\end{aligned}
$$

Queste ultime relazioni sviluppate scrivonsi:

$$
\begin{aligned}
e^{-\theta}\left(b e^{\theta}-a e^{\gamma}+a^{2} b e^{-\varphi}\right. & \left.-a b^{2} e^{--}\right)+e^{\theta}\left(a e^{-\lambda}-b e^{-\varphi}\right) \\
& =e^{-\psi}\left(a e^{\varphi}-b e^{\lambda}+a b^{2} e^{-\phi}-a^{2} b e^{-\lambda}\right)+e^{\psi}\left(b e^{-\lambda}-a e^{-\phi}\right), \\
e^{-\theta}\left(b e^{\varphi}-a e^{\lambda}-a^{2} b e^{-\phi}\right. & \left.+a b^{2} e^{-\gamma}\right)+e^{\theta}\left(a e^{-\lambda}-b e^{-\phi}\right) \\
& =e^{-\psi}\left(a e^{\phi}-b e^{\lambda}-a b^{2} e^{-\phi}+a^{2} b e^{-\lambda}\right)+e^{\psi}\left(b e^{-\lambda}-a e^{-\phi}\right)
\end{aligned}
$$

e per somma e differenza forniscono le altre due:

$$
\begin{aligned}
e^{-\theta}\left(b e^{\varphi}-a e^{\lambda}\right)+e^{\dagger}\left(a e^{-\lambda}-b e^{-\varphi}\right) & =e^{-\psi}\left(a e^{\varphi}-b e^{\lambda}\right)+e^{\psi}\left(b e^{-\lambda}-a e^{-\phi}\right) \\
e^{-\theta}\left(a^{2} b e^{-\varphi}-a b^{2} e^{-\lambda}\right) & =e^{-\psi}\left(a b^{2} e^{-\varphi}-a^{2} b e^{-\lambda}\right) .
\end{aligned}
$$

L'ultima di queste da intanto:

$$
e^{\psi-\theta}=\frac{b e^{-\varphi}-a e^{-\lambda}}{a e^{-\phi}-b e^{-\lambda}}=\frac{b e^{\gamma}-a e^{\varphi}}{a e^{j}-b e^{\phi}}
$$


ed in forza di questa, l'altra dat concordemente:

$$
e^{\psi-\theta}=\frac{a e^{\varphi}-b e^{\lambda}}{b e^{\varphi}-a e^{\lambda}} .
$$

Concludiamo allora che se effettivamente esiste un integrale $\psi$ comune ai due sistemi $\Phi(\varphi, \psi ; b), \Phi(\lambda, \psi ; a)$ esso è necessariamente della forma;

$$
\psi=\theta+\log \frac{a e^{\varphi}-b e^{\hat{\gamma}}}{b e^{\varphi}-a e^{j}} .
$$

Noi verificheremo che tale espressione è effettivamente un integrale comune ai si-

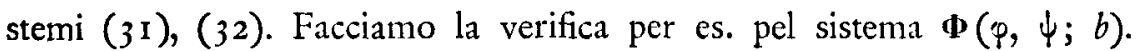

Intanto si ha:

$$
\begin{gathered}
\frac{\partial \varphi}{\partial u}+i \frac{\partial \psi}{\partial v}=\frac{\partial \psi}{\partial u}+i \frac{\partial \theta}{\partial v}+i \frac{a e^{\varphi} \frac{\partial \varphi}{\partial v}-b e^{2} \frac{\partial \lambda}{\partial v}}{a e^{\varphi}-b e^{\lambda}}-i \frac{b e^{\varphi} \frac{\partial \varphi}{\partial v}-a e^{\lambda} \frac{\partial \lambda}{\partial v}}{b e^{\varphi}-a e^{\lambda}} \\
=A(\varphi, \theta, a)+\left[B(\varphi, \theta, a)-\frac{\partial \theta}{\partial u}\right]\left(\frac{a e^{\varphi}}{a e^{\phi}-b e^{\lambda}}-\frac{b e^{\varphi}}{b e^{\varphi}-a e^{\lambda}}\right)- \\
-\left[B(\lambda, \theta, b)-\frac{\partial \theta}{\partial u}\right]\left(\frac{b e^{\lambda}}{a e^{\varphi}-b e^{\lambda}}-\frac{a e^{\lambda}}{b e^{\varphi}-a e^{\lambda}}\right) \\
=A(\varphi, \theta, a)+B(\varphi, \theta, a)\left(\frac{a e^{\phi}}{a e^{\varphi}-b e^{\lambda}}-\frac{b e^{\phi}}{b e^{\varphi}-a e^{\lambda}}\right)- \\
-B(\lambda, \theta, b)\left(\frac{b e^{\lambda}}{a e^{\varphi}-b e^{\lambda}}-\frac{a e^{\lambda}}{b e^{\varphi}-a e^{\lambda}}\right)=A(\varphi, \psi, b)
\end{gathered}
$$

e ciò dimostra intanto che $\psi$ è soluzione della prima equazione del sistema (3I).

In modo perfettamente analogo si verifica che $\psi$ è soluzione della seconda equazione del medesimo sistema come pure che essa è soluzione del sistema $\Phi(\lambda, \psi ; a)$.

Diciamo quindi:

Se $\theta(u, v)$ è soluzione comune ai due sistemi $\Phi(\varphi, \theta ; a), \Phi(\lambda, \theta ; b)$ la funzione

$$
\psi=\theta+\log \frac{a e^{\varphi}-b e^{2}}{b e^{\varphi}-a e^{\lambda}}
$$

$\dot{e}$ soluzione comune ai due sistemi $\mathbf{\Phi}(p, \psi ; b), \mathbf{\Phi}(\lambda, \psi ; a)$.

18. Vediamo quale significato ha il precedente teorema per le trasformazioni di Darboux. Unitamente ai sistemi del $\mathrm{n}^{\circ}$ precedente consideriamo le quattro superficie minime $S_{\theta}, S_{\varphi}, S_{\lambda}, S_{\psi}$; si hanno le relazioni di trasformazione:

$$
\begin{array}{ll}
S_{\varphi} \cdot D_{a} \equiv S_{\theta}, & S_{\lambda} \cdot D_{b} \equiv S_{\theta}, \\
S_{\varphi} \cdot D_{b} \equiv S_{\psi}, & S_{\lambda} \cdot D_{a} \equiv S_{\psi}
\end{array}
$$

onde risulta il seguente teorema $d_{1}$ permutabiliti: 
Se $S_{\theta}$ è una superficie minima contigua per trasformazioni di Darboux $D_{a}, D_{b} a$ due superficie minime $S_{\varphi}, S_{\lambda}$ esiste una quarta superficie minima $S_{\psi}$, perfettamente determinata, contigua alle $S_{\varphi}, S_{\lambda}$ per trasformazioni di Darboux $D_{b}, D_{a}$ a costanti permutate.

Questo notevole teorema è del tutto analogo a quello che si ha per le trasformazioni $B_{\sigma}$ delle superficie a curvatura totale costante.

E superfluo avvertire che dalla $S_{\theta}$ si passa alla $S_{\downarrow}$ operando una trasformazione $D_{-a}$ e poi una $D_{b}$ ovvero una $D_{-b}$ e poi una $D_{a}$; di tale circostanza bisogna tener conto quando si vogliono applicare le formule di composizione stabilite al $\mathrm{n}^{\circ}$ I6.

$\mathrm{Si}$ verificherà facilmente che le espressioni di $A, B, C ; M, N$ allora trovate rimangono in loro stesse operando la sostituzione:

e ponendo:

$$
\Sigma \equiv\left(\begin{array}{lll}
a, & b, & \varphi \\
b, & a, & \lambda
\end{array}\right)
$$

$$
\psi=\theta+\log \frac{a e^{\varphi}-b e^{\lambda}}{b e^{\varphi}-a e^{\lambda}} .
$$

Sulle quattro superficie $S_{\theta}, S_{\phi}, S_{\lambda}, S_{\psi}$ si considerino ordinatamente quattro punti corrispondenti $P_{\mathrm{I}}, P_{2}, P_{3}, P_{4}$; ricordando le proprietd stabilite al $\mathrm{n}^{\circ} \mathrm{I} 8$, si ha che:

Al variare della quaderna $P_{1}, P_{2}, P_{3}, P_{4}$ di punti corrispondenti il quadrilatero che ba per vertici questi punti conserva invariate le lungbezze dei lati e delle diagonali.

19. Dal teorema di permutabilita dimostrato possonsi dedurre conseguenze analoghe a quelle che si hanno per le superficie a curvatura totale costante.

Cosi per es. supponiamo che della superficie minima $S_{\theta}$ si conoscano tutte le trasformate di Darboux, si sappia cioè integrare il sistema $\Phi(\theta, \varphi ; t)$ per qualunque valore della costante $t$ (la quale peraltro, data la definizione della trasformazione, è diversa da zero). Si conoscerà allora una soluzione:

$$
\varphi=\varphi(u, v, t, c)
$$

con due costanti arbitrarie $t, c$ della equazione:

$$
\frac{\partial^{2} \varphi}{\partial u^{2}}+\frac{\partial^{2} \varphi}{\partial v^{2}}=e^{-2 \varphi}
$$

allora si puó dimostrare che:

Per ciascuna superficie $S_{\lambda}$ contigua per certa trasformazione $D_{a}$ alla $S_{\theta}$ le trasformate di Darboux si determinano con soli calcoli algebrici e di derivazione.

Infatti sia:

$$
\lambda=\varphi(u, v, a, c)
$$

la soluzione del sistema $\Phi(\theta, \lambda ; a)$ corrispondente a $S_{\lambda}$; se $S_{\varphi}$ è la trasformata generica per trasformazionc $D_{t}$ di $S_{f}$, il teorema di permutabiliti, per la funzione $\mu$. cor- 
rispondente alla trasformata generica $S_{\mu}$ di $S_{\lambda}$ ci di:

$$
\mu=\theta+\log \frac{a e^{\varphi(u, v, l, c)}-t e^{\lambda}}{t e^{\varphi(u, v, t, c)}-a e^{\lambda}}
$$

onde in termini finiti deducesi la trasformata $S_{\mu}$ di $S_{\lambda}$.

Evidentemente peró questo procedimento di trasformazione non ha l'interesse che il procedimento analogo ha per le superficie a curvatura totale costante in quanto che l'equazione di Lrouville, per le superficie minime si sa integrare e quindi, com'è noto, si conoscono gid tutte le superficie d'area minima.

20. Dalle cose esposte appare chiaramente l'analogia che esiste fra le trasformazioni $D_{t}$ delle superficie minime e le trasformazioni $B_{\sigma}$ delle superficie a curvatura totale costante. Ma le trasformazioni $D_{t}$ ora studiate pare debbano avere interesse più grande se si ricollegano alle classi complete di superficie applicabili deducibili in modo noto dalle superficie minime.

Il BiAnCHI ha gid mostrato ciò nella nota citata e in quegli studî appare probabile che le trasformazioni gia note delle superficie minime debbansi risolvere in successive trasformazioni più semplici per le quali notevole importanza devono avere le trasformazioni $D_{t}$, similmente a quello che accade per le trasformazioni di BäckLund delle superficie pseudosferiche le quali risolvonsi sempre nel prodotto di una trasformazione di Branchi e di una trasformazione di LiE.

22. I casi in cui due superficie $S, S^{\prime}$ possono essere vincolate per configurazioni invariabili sono stati passati in rassegna in modo, crediamo, abbastanza soddisfacente e completo. Da tutti i casi studiati emerge infine il teorema:

Una trasformazione per configurazioni invariabili conserva le linee di curvatura.

$\mathrm{E}$ tale circostanza che ci ba indotto a scegliere a sistema coordinato sulla super-. ficie di partenza appunto quello delle linee di curvatura le formule e gli sviluppi assumendo allora aspetto relativamente semplice.

Palermo, ottobre 1921.

PiETRO TORTORICI. 